\title{
Time-Variant Reliability Assessment through Equivalent Stochastic Process Transformation
}

\author{
Zequn Wang ${ }^{1}$ and Wei Chen ${ }^{2}$ \\ Department of Mechanical Engineering, Northwestern University, Evanston, IL 60208, USA
}

\begin{abstract}
Time-variant reliability measures the probability that an engineering system successfully performs intended functions over a certain period of time under various sources of uncertainty. In practice, it is computationally prohibitive to propagate uncertainty in time-variant reliability assessment based on expensiv or complex numerical models. This paper presents an equivalent stochastic process transformation approach for cost-effective prediction of reliability deterioration over the life cycle of an engineering system. To reduce the high dimensionality, a time-independent reliability model is developed by translating random processes and time parameters into random parametersin order to equivalently cover all potential failures that may occur during the time interval of interest. With the timeindependent reliability model, an instantaneous failure surface is attained by using a Kriging-based surrogate model to identify all potential failure events. To enhance the efficacy of failure surface identification, a maximum confidence enhancement method is utilized to update the Kriging model sequentially. Then, the time-variant reliability is approximated using Monte Carlo simulations of the Kriging model where system failures over a time interval are predicted by the instantaneous failure surface. The results of two case studies demonstrate that the proposed approach is able to accurately predict the time evolution of system reliability while requiring much less computational efforts compared with the existing analytical approach.
\end{abstract}

Key words: random process, Kriging, reliability analysis, adaptive sampling, time-variant

${ }^{1}$ Postdoctoral Fellow, zequn.wang@ northwestern.edu

${ }^{2}$ Professor, wei.chen@ northwestern.edu, Corresponding Author 


\section{NOMENCLATURE}

$\begin{array}{lll}R & = & \text { reliability } \\ \Phi & = & \text { standard Gaussian cumulative distribution function } \\ \beta_{\mathrm{t}} & = & \text { target reliability index } \\ E I & = & \text { expected improvement } \\ f_{x}(x) & = & \text { probability density function } \\ f(\bullet \mid \bullet) & = & \text { conditional probability density function or likelihood function } \\ p_{f} & = & \text { probability of failure } \\ I_{f} & = & \text { indicator function } \\ P_{f} & = & \text { probability of failure } \\ \lambda(t) & = & \text { out-crossing rate } \\ \mathbf{R} & = & \text { correlation matrix } \\ \mathbf{C o v} & = & \text { covariance matrix } \\ \mathbf{M} & = & \text { GP model } \\ T_{f} & = & \text { time to failure }\end{array}$

\section{INTRODUCTION}

For decades, time-invariate reliability methods, such as the most probable point based methods [1, 2], univariate dimension reduction method [3-5], polynomial chaos expansion [6-8] and surrogate-based methods [9-11], have been well developed to approximate probability of failure without the consideration of stochastic aging effects. As an engineered system ages under stochastic operational conditions, system failures tend to occur, which may lead to catastrophic consequences. As a result, the aggregated risk in operating an engineering system over time is not controllable which substantially increases the lifecycle cost even though a high initial reliability level of the engineered system is ensured using the aforementioned time-invariant reliability tools. In recent years, time-variant reliability analysis has drawn vast research efforts to ensure a high reliability level of an engineered system during its life cycle. In order to tackle the aging effect, time parameter is often considered in modeling the performance of engineered 
systems either directly as an input or indirectly as random processes. The stochastic operational conditions characterized as random processes can be a typical example of introducing time parameter. In time-variant reliability analysis, various sources of uncertainties are propagated to the time-dependent response for quantitatively assessing the probability that the system can successfully realize the intended functions over a time period. Due to the prohibitively high computational cost for uncertainty propagation using time-consuming numerical simulations directly, it is still a grand challenge for conducting time-variant reliability assessments in an efficient and accurate manner.

To handle time dependency of system failures, various approaches have been developed, which can be generally categorized into two types, the first-passage based approaches [12-18] and the extreme value based approaches [19-23]. In the first-passage based approaches, outcrossing events will occur if the system performance exceeds the upper bound or falls below the lower bound of the safety threshold, and thus the first order derivative of reliability with respect to time can be approximated by an out-crossing rate measure. With an assumption that all outcrossing events are independent, the time-variant probability of failure is approximated by an integration of the out-crossing rate. As a representative of first-passage based methods, the PHI2 approach [13] computes the instantaneous probability of failures in the U-space using the firstorder reliability method (FORM), and then approximates the out-crossing rate based on the reliability indices of two successive time nodes and the autocorrelation of system responses. Even though the first-passage based methods tend to derive the time-variant reliability analytically and thus are mathematically sound, the accuracy of time-variant reliability assessment is hampered by approximations in the embedded FORM algorithm and the low resolution of discretized time dimension. Moreover, the error is often significant for the cases of 
large probability of failure as the underlying assumption of independent crossing events does not hold. In addition, an optimization problem is solved iteratively by searching the most probability point (MPP) at each discretized node across time scale, which makes the time-variant reliability assessment computationally expensive. In the extreme value based approaches, the worst scenarios of system performances over time scale are extracted as a measure to quantify the reliability. To do so, the extreme value distribution of a critical system performance is first approximated by propagating an input variability to the performance of a dynamic system. A failure will occur if the extreme value of the performance does not satisfy system requirements. With the extreme value distribution, time-invariant reliability tools are readily applied for calculating time-variant reliability. Nevertheless, it is technically difficult to derive a close-form solution of the extreme value and it becomes computationally prohibitive to obtain the distribution of extreme performances using sampling approaches such as Monte Carlo simulation (MCS). In practice, expensive numerical models are usually employed to implicitly evaluate the limit state function, which makes reliability assessment computationally prohibitive. To overcome the computational burden, surrogate models, such as polynomial chaos expansion [68], Kriging [19, 21, 24], and artificial neural networks [25] are widely employed to replace the original expensive simulation in reliability analysis $[26,27]$. As an example, the nested extreme response surface (NERS) approach [21] constructs a response surface over an input space to predict the critical time when the engineered system reaches the extreme performance over a time period. Thus, the worst scenarios are predicted by the response surface, which converts time-variant reliability problems to time-independent counterparts and enables the integration of existing advanced time-invariant reliability tools into time-variant reliability approximation. Even though NERS greatly alleviates the computational burden of time-variant reliability 
analysis, one major disadvantage lies in the lack of capability of propagating random processes to dynamic responses of engineered systems. In this work, an equivalent stochastic process transformation approach (eSPT) is proposed for time-variant reliability assessment, with an aim to significantly reduce the extremely high computational cost which is a major challenge confronted by both out-crossing rate based methods and extreme value based methods. It is worth noting that eSPT dose not intend to approximate stochastic process by random parameters. By equivalent, we mean that the resulting failure domained after equivalent stochastic process transformation is able to cover all the instantaneous failure events during a certain time period. Different from discretizing time scale for handling random process inputs in the existing approaches, the eSPT incorporates time as another uniformly distributed input parameter and transforms the random processes into equivalent random variables. Thus an instantaneous reliability model is constructed to envelope all potential system failures that may occur in operating dynamic systems over time. The maximum confidence enhancement method is employed to accurately identify the instantaneous failure surface, by which the time-variant reliability is approximated using MCS. The novelty of eSPT lies in the significant reduction of the dimensionality of time-variant reliability problems by the translation of random processes and time parameters, and by the prediction of time-variant failure events based on the instantaneous failure surface.

The benefits of using sSPT over existing time-variant reliability approaches are multiple folds. On one hand, the accuracy and efficiency of existing time-variant reliability approaches highly depend on the magnitude of time step for discretizing time scale. Higher accuracy level will be attained with a smaller time step, but that may require much more computational resources. In contrast, the eSPT treats time and random processes as additional continuous 
random variables in constructing the instantaneous failure surface. Once an accurate instantaneous failure surface is obtained, extra computational cost is not required no matter how small the time step adopted in MCS is. On the other hand, the eSPT constructs an instantaneous reliability model to cover the important region where the time-dependent system failure may occur, and thus enables the use of existing time-invariant reliability tools for predicting timevariant reliability. In addition, a confidence-based surrogate modeling approach is adopted as a time-invariant reliability tool to actively learn the instantaneous failure surface, and thus to enhance the performance of eSPT for time-variant reliability assessment. The rest of the paper is organized as follows. The existing time-variant reliability approaches are reviewed in Section 2. Section 3 describes the proposed equivalent stochastic process approach. Two case studies are used to demonstrate the effectiveness of the developed methodology in Section 4.

\section{REVIEW OF TIME-VARIANT RELIABILITY ANALYSIS METHODS}

In this section, we provide the general definition of time-variant reliability and briefly review the existing approaches in literature. In practice, the performance of engineered systems are often dynamic and thus a function of time. A system failure occurs if the time-dependent performance function does not satisfy the requirement at any instant during a certain period of time. $\operatorname{Let} \mathrm{g}(\mathbf{X}$, $\mathbf{Y}(t), t)$ denotes the limit state function, where $\mathbf{X}=\left[\mathrm{X}_{1}, \mathrm{X}_{2}, \ldots, \mathrm{X}_{n}\right]$ is a vector of $n$ random variables and $\mathbf{Y}(t)=\left[\mathrm{Y}_{1}(t), \mathrm{Y}_{2}(t), \ldots, \mathrm{Y}_{m}(t)\right]$ is a vector of $m$ input random processes with the time $t$. For a specified time period $\left[0, T_{L}\right]$, system failure occurs if there is a time node $t$ in $\left[0, T_{L}\right]$ such that the limit state value is greater than zero, which is expressed by

$$
\exists t \in\left[0, T_{L}\right], g(\mathbf{X}, \mathbf{Y}(t), t)>0
$$

It is worth noting that the failure event might be defined with $g()<$.0 in the literature. Thus the probability of failure over a time interval $[0, T]\left(0 \leq T \leq T_{L}\right)$ is a monotonic increasing function with 
$T$ and can be calculated by

$$
P_{f}(0, T)=\operatorname{Pr}(\exists t \in[0, T], g(\mathbf{X}, \mathbf{Y}(t), t)>0), 0 \leq T \leq T_{L}
$$

As a result, the time-variant reliability is obtained by

$$
R(0, T)=1-P_{f}(0, T), 0 \leq T \leq T_{L}
$$

It is technically intractable in deriving a closed-form solution for calculating time-variant probability of failure in Eq. (2) due to the time-dependency of system failures. Various approaches have been developed in the literature to obtain an approximation of time-variant reliability. Here we briefly review two kinds of wildly used approaches in the literature.

\subsection{Out-crossing Rate Based Methods}

In the context of time-variant reliability, out-crossing rate based methods were developed based on the concept of out-crossing events. A crossing event occurs when the time-dependent performance of an engineered system goes beyond an upper limit or falls below a lower limit, which makes a transition of engineered system from success state to failure state. Time-variant reliability analysis is then performed by analyzing crossing rates with respect to time.

By assuming that all crossing events are independent, the crossing rate at time instant $t$ is derived by the limit of a conditional probability as

$$
\lambda(t) \approx \lim _{\Delta t \rightarrow 0} \frac{\operatorname{Pr}\{g(\mathbf{X}, \mathbf{Y}(t), t)>0 \bigcap g(\mathbf{X}, \mathbf{Y}(t+\Delta t), t+\Delta t)<0\}}{\Delta t} .
$$

Then, the probability of failure over the time interval $[0, T]$ is calculated as

$$
P_{f}(0, T)=1-\left(1-P_{f}(0,0)\right) \exp \left\{-\int_{0}^{T} \lambda(t) \mathrm{d} t\right\}
$$

where $P_{f}(0,0)$ denotes the time-invariant probability of failure at the initial time $T=0$. Many researchers have developed advanced algorithms for approximating the crossing rate for general 
stochastic processes. Among the existing extensive studies, Rice formula [21] has been widely employed to compute the crossing rate based on the First-Order Reliability Method (FORM) [2]. To calculate the crossing rate, the limit state function is first discretized by a small time step and thus translate the random processes $\mathbf{Y}(t)$ to random variables at each time node. Then, the FORM is employed to compute the reliability index at each time node. In FORM, the limit state function at a given time node is transformed into a standard normal space, in which the most probable point (MPP) is iteratively searched using an optimization algorithm. The FORM is repeatedly utilized to obtain reliability index at all time nodes, then the crossing rate is approximated by the reliability indexes of two successive time notes and by the correlation of the input stochastic process $\mathbf{Y}(t)$. Though a mathematical formulae of the time-variant reliability is derived rigorously, the analytical form of the crossing rate is still technically intractable for general stochastic processes in practice. In addition, the accuracy of time-variant reliability analysis depends on the resolution of discretized time scale and significant errors may be induced by the FORM algorithm in computing time-invariant reliability indices.

\subsection{Extreme Value Based Methods}

In the extreme value based methods, the worst scenarios of an engineered system over a specified period of time are extracted as a measure for time-variant reliability analysis. Given that $g(\mathbf{X}, \mathbf{Y}(t), t)>0$ indicates failure, the worst system performance is obtained by maximizing the time-dependent limit state function within a time period $[0, T]$, which is described as

$$
g_{e}(\mathbf{X}, T)=\max _{t \in[0, T]} g(\mathbf{X}, \mathbf{Y}(t), t),
$$

where $g_{e}$ represents the extreme value and is a random variable due to the randomness of input parameters $\mathbf{X}$ and $\mathbf{Y}(t)$. Thus, failure events are exactly determined by the extreme value and the probability of failure over the time period $[0, T]$ is derived as 


$$
P_{f}(0, T)=\operatorname{Pr}\left(g_{e}(\mathbf{X}, T)>0\right) .
$$

It is difficult to analytically obtain the probability characteristics of the extreme value $g_{e}$ for complex engineering applications, thus simulation-based methods can be used as an alternative way to approximate the probability of failure in Eq. (7). The approximation of time-variant reliability can be very accurate using simulation-based methods such as Monte Carlo simulation (MCS), however, it is computationally expensive to apply directly to the expensive simulations as a large number of evaluations are often required.

To alleviate the computational burden, the nested extreme response surface (NERS) method [21] was developed to construct a time response surface, by which the worst scenarios of system performances during a certain period of time can be efficiently extracted. With an underlying assumption that the time-dependent limit state function is an explicit function of random variable $\mathbf{X}$ and time $t$, the response surface of time corresponding to the worst scenarios is given by

$$
T_{e}(\mathbf{X})=\left\{t \mid \max _{t} g(\mathbf{X}, t), t \in[0, T]\right\}
$$

Then the probability of failure during the time interval $[0, T]$ is derived as

$$
P_{f}(0, T)=\operatorname{Pr}\left(g_{e}>0\right)=\operatorname{Pr}\left(g\left(\mathbf{X}, T_{e}(\mathbf{X})\right)>0\right)
$$

With the response surface of time in NERS, FORM is directly employed as a reliability tool to calculate time-variant reliability in Eq. (9). Though NERS demonstrates significant improvements in efficiency, the major limitation of the NERS lies in its lack of capability in handling random process parameters $\mathbf{Y}(t)$. In Section 3, we introduce the proposed equivalent stochastic process transformation approach for handling all kinds of aleatory uncertain system inputs and performing time-variant reliability assessment in an efficient and accurate manner.

\section{EQUIVALENT STOCHASTIC PROCESS TRANSFORMATION APPROACH}


This section presents the proposed equivalent stochastic process transformation approach (eSPT) for time-variant reliability assessment. The concept of random field transformed space failure envelop is first introduced in Section 3.1 while Section 3.2 presents the instantaneous reliability model using a translation of time and random processes parameters. Section 3.3 describes the surrogate modeling technique for predicting instantaneous failure events. The maximum confidence enhancement method is elaborated in Section 3.4 to enhance the fidelity of surrogate models from the perspective of probability predictions. The approximation of timevariant reliability using MCS is detailed in Section 3.5.

\subsection{Random Field Transformed Space}

In the context of time-invariant reliability analysis, a failure occurs if a time-independent limit state function goes beyond a predefined allowable threshold. Nevertheless, the limit state function is time-dependent for dynamic systems. For such systems, a failure is defined accordingly as if there is any time instant within a specified period of time $\left[0, T_{L}\right]$ that the failure occurs. Thus one failure in time-variant reliability analysis may contain several failure events at multiple time nodes across time scale. In this research, we introduce the concept of failure envelope to cover all the potential failure events which will be assembled to predict time-variant failures efficiently.

In a broad sense, the time-dependent limit state function is a complex stochastic process with random inputs $\mathbf{X}$ and random processes $\mathbf{Y}(t)$. Charactering the probabilistic properties of extreme value distribution of $g(\mathbf{X}, \mathbf{Y}(t), t)$ usually demands vast computational resources, which significantly impedes the practical use of the extreme-value based approaches. In Figure 1, a realization of the time-dependent limit state function is plotted. Clearly, there are two crossing events occurred at time instant $t_{1}$ and $t_{3}$ while the worst performance exists within the time 
interval $\left[t_{1}, t_{2}\right]$. Within the time intervals $\left[t_{1}, t_{2}\right]$ and $\left[t_{3}, t_{4}\right]$, instantaneous failures occur. Different from the existing time-variant reliability approaches, the eSPT does not involve the calculation of extreme performances and identification of crossing events. In contrast, the instantaneous failure events are identified efficiently and used to predict time-variant reliability in the eSPT.

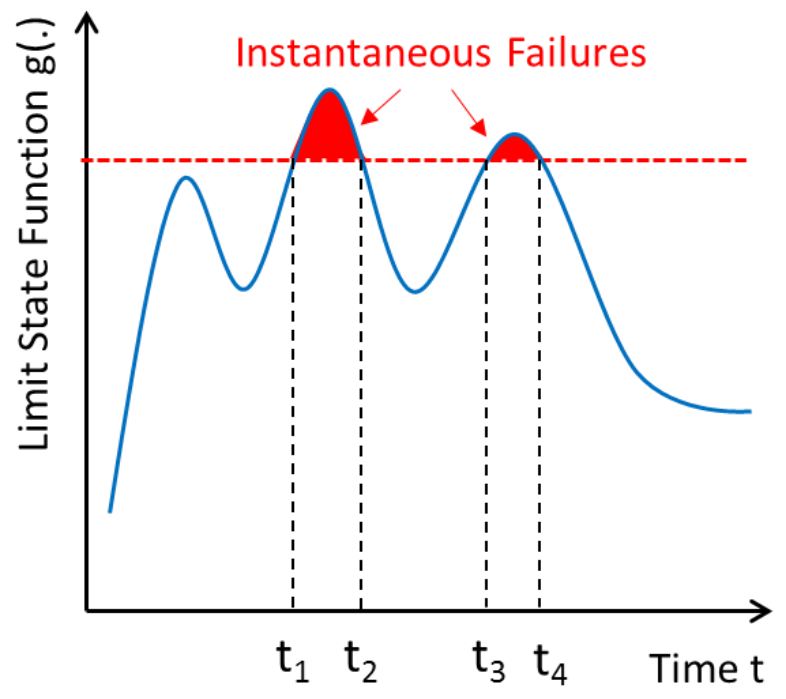

Figure 1: Time-dependent Limit State Function and Instantaneous Failures

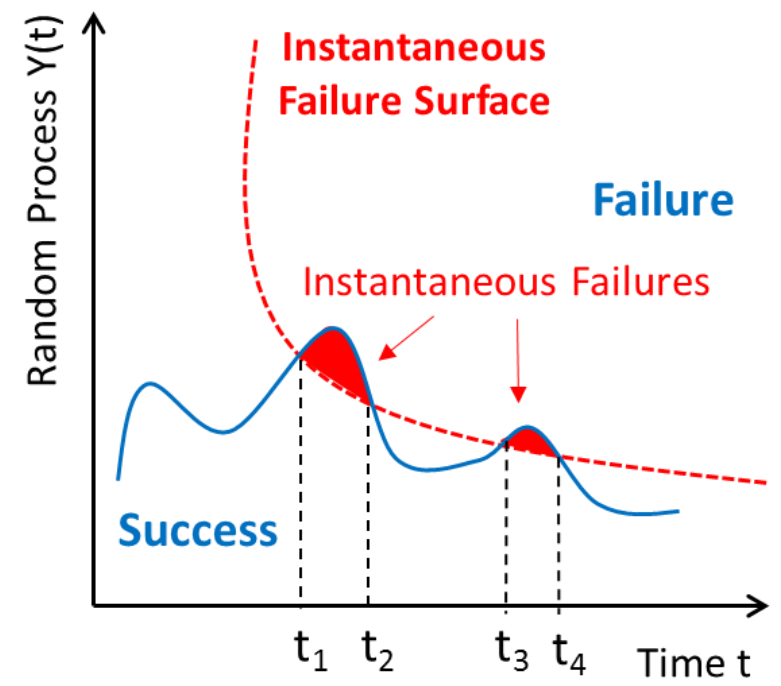

Figure 2: Random Field Transformed Space

To identify all instantaneous failures, the time-dependent limit state function is transformed to 
a new input space, referred to as "random field transformed space", in which time $t$, random variables $\mathbf{X}$ and random process parameters $\mathbf{Y}(t)$ are treated as continuous variables. In the random field transformed space, one time-series realization of multi-variant random process system inputs is a one-dimensional curve. The blue solid curve in Figure 2 is an illustration example of the multi-variant random process when there is one random parameter $\mathbf{X}$ and only one random process $\mathbf{Y}(t)$. In the random field transformed space, the original time-variant reliability problem is translated to a typical time-invariant counterparts to identify instantaneous failure events, which enables the prediction of time-variant failure events using MCS without extra computational cost. In the next subsection, an instantaneous reliability model will be constructed in the random field transformation space to cover all potential instantaneous failure events that may occur for dynamic system during a specified period of time.

\subsection{Instantaneous Reliability Model}

After a transformation of the time-dependent limit function, the instantaneous failure event at a certain time instant $t$ is defined in the random field transformed space as

$$
g\left(\mathbf{X}^{\prime}, \mathbf{Y}^{\prime}, t^{\prime}\right)>0
$$

where $\mathbf{X}^{\prime}, \mathbf{Y}^{\prime}$, and $t^{\prime}$ are continuous variables translated from random parameters $\mathbf{X}$, random processes $\mathbf{Y}(t)$ and time $t$. The translated limit state function $g\left(\mathbf{X}^{\prime}, \mathbf{Y}^{\prime}, t^{\prime}\right)$ possesses the capability of identifying all potential instantaneous failure events for predicting time-variant failures, and thus for assessing time-variant reliability of dynamic systems. Nevertheless, it is computationally prohibitive and unnecessary to learn the instantaneous failure surface in a global sense using simulate-based methods. In principle, a critical local area that encompasses all potential instantaneous failure events should be identified given the probabilistic characterization of the system inputs $\mathbf{X}$ and $\mathbf{Y}(t)$. Thus, an instantaneous reliability problem is formulated in the random 
field transformed space such that the resulting failure area is able to cover all the instantaneous failure events in the corresponding time-variant reliability assessment.

In order to accurately obtain the instantaneous failure surface in the critical local region, this work constructs an instantaneous reliability model based on a translation of random system's inputs. The instantaneous failure surface is then obtained by solving the instantaneous reliability model using time-invariant reliability tools. In the instantaneous reliability model, the randomness of input parameters should be carefully designed such that the resulting instantaneous failure surface can cover the relevant critical region for predicting time-variant failures. To do so, a translation of system random inputs and time parameter is developed in this section. As shown in Figure 3, the random process $\mathbf{Y}(t)$ becomes a random parameter at any time node, and the probability density function (PDF) of $\mathbf{Y}(t)$ at a certain time node is obtained accordingly based on the given probabilistic information of $\mathbf{Y}(t)$. Thus, the probability density function of $\mathrm{Y}^{\prime}$ is obtained by an average of the PDFs of random process $\mathbf{Y}(t)$ over time, which is expressed as

$$
f_{p d f}\left(\mathbf{Y}^{\prime}\right) \approx \sum_{i=1}^{s} f_{p d f}\left(\mathbf{Y}\left(t_{i}\right)\right),
$$

where $f_{p d f}($.$) is the probability density function, t_{i}$ is the $i^{\text {th }}$ time node and $s$ represents the total number of time nodes for discretizing random processes $\mathbf{Y}(t)$. It is worth noting that obtaining the probability density function of $\mathbf{Y}^{\prime}$ is very cheap compared with performance evaluations of dynamic systems, thus one can always obtain an accurate approximation of the PDF of $\mathbf{Y}^{\prime}$ by increasing the number of time nodes $s$. For some specific cases such as Gaussian processes, the distribution information of $\mathbf{Y}^{\prime}$ can be analytically computed. For general cases, the random realizations of random process $\mathrm{Y}(\mathrm{t})$ are generated based on spectral decomposition and the distribution information of $\mathbf{Y}^{\prime}$ is readily approximated. 


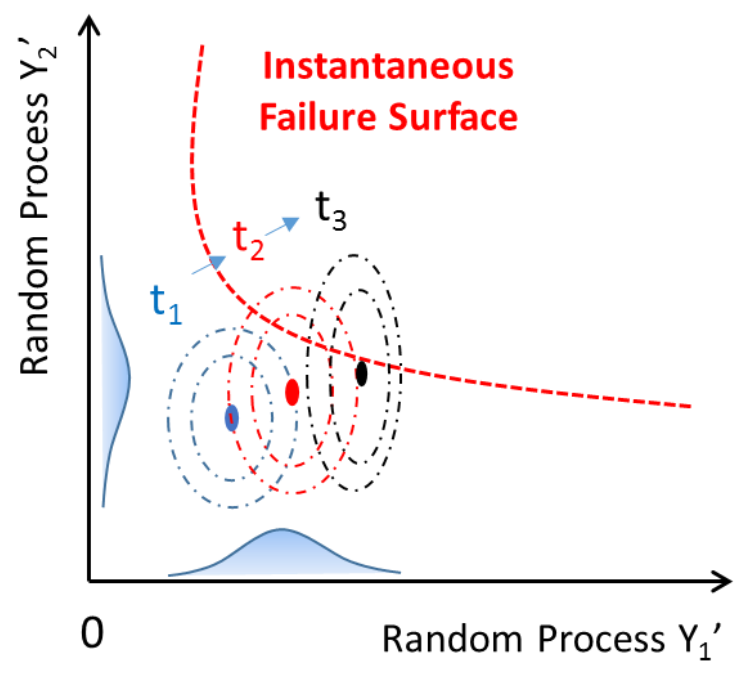

Figure 3: Transformation of Random Processes $\mathbf{Y}(t)$

The translation of the random variable $\mathrm{X}$ and time parameter $\mathrm{t}$ is graphically shown in Figure 4. As the random variables $\mathbf{X}$ are time independent, the distributions of $\mathbf{X}$ remain the same over time. Thus, there is no need to perform a transformation for random variables $\mathbf{X}$ in the instantaneous reliability model. For the parameter time, let $T_{L}$ denotes the life cycle of dynamic systems, a uniform distribution on an interval $\left[0, T_{L}\right]$ is assigned for the parameter $t$ '. The uniform distribution is employed for time parameter because the instantaneous failure events at different time are equally important. The instantaneous reliability model is then constructed for the time-variant reliability analysis as

$$
P_{f}=\operatorname{Pr}\left(g\left(\mathbf{X}, \mathbf{Y}^{\prime}, t^{\prime}\right)>0\right),
$$

where $P_{f}$ is the probability of failure, $\mathbf{X}$ are the random parameters defined for time-variant reliability analysis, $\mathbf{Y}^{\prime}$ are random parameters transformed from the random processes $\mathbf{Y}(t)$, and $t^{\prime}$ is a uniformly distributed random variable within an interval $\left[0, T_{L}\right]$. By solving Eq. (12), an instantaneous failure surface is approximated using time-invariant reliability tools, and then utilized for predicting time-variant reliability using MCS. It is worth noting that the goal of random processes transformation is to reduce the dimensionlity of time-variant reliability 
problems in a way that the resulting failure domain after transformation is equivalent with the one formulated by the original time-variant reliability problem.

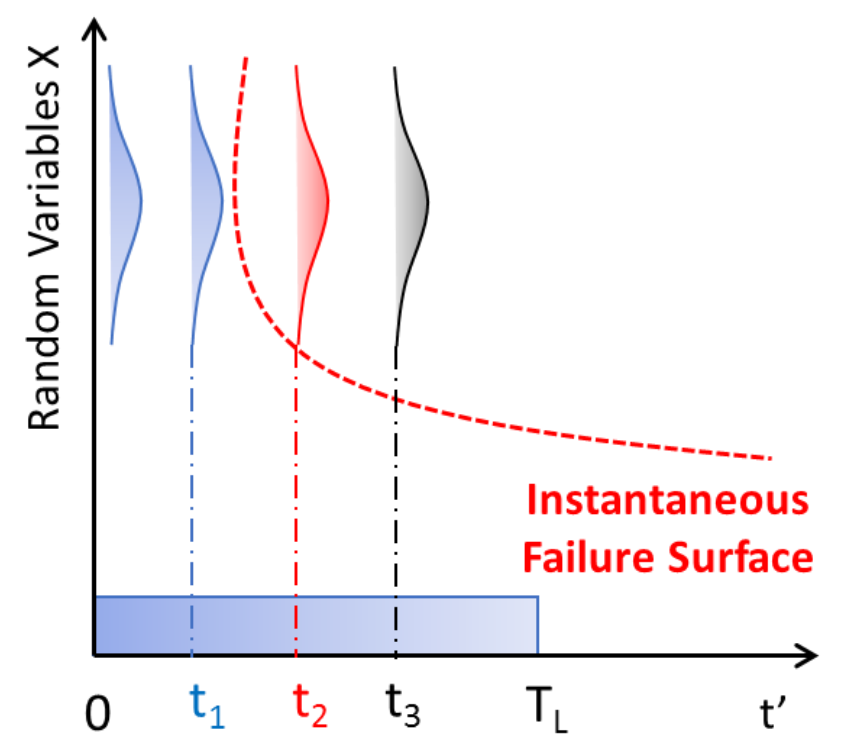

Figure 4: Transformation of Random Variable X and Time Parameter $t$

\subsection{Limit State Surrogate Modeling}

With the constructed instantaneous reliability model, time-invariant reliability tools, such as the first-order reliability method (FORM), polynomial chaos expansion (PCE) method, and Kriging-based methods, are capable of calculating the probability of failure in Eq. (12). FORM simplifies the limit state function at the most probable point in the standard normal space, and thus may lack the ability of predicting the instantaneous failure events for nonlinear cases. PCE is able to construct a response surface for accurately learning the limit state function with sufficient orthogonal polynomials in the expansion while requiring vast computational resources. In addition, it is still technically difficult for PCE to adaptively update the surrogate model in a local critical area. To address this, this work employs Kriging technique to initially construct a low fidelity surrogate model for the limit state function $g\left(\mathbf{X}, \mathbf{Y}^{\prime}, t^{\prime}\right)$, and then enhance the fidelity 
of instantaneous failure surface using an adaptive sampling approach.

Kriging method has been widely applied for building surrogate models in strucutural reliability analysis $[9,19,21,28-31]$. The method is capable of quantifying epistemic uncertainty due to the lack of data in constructing surrogate models, which enables the further development of adaptive sampling techniques. For incorporating adaptive sampling techniques in time-variant reliability assessments, a novel equivalent stochastic process transformation is developed in this paper. In the following, Kriging modeling is introduced in this section while Section 3.4 presents the recently developed maximum confidence enhancement based adaptive sampling scheme by author [9]. Let $\mathbf{W}=\left[\mathbf{X}, \mathbf{Y}^{\prime}, t^{\prime}\right]$ denote the input parameters of the limit state function $g\left(\mathbf{X}, \mathbf{Y}^{\prime}, t^{\prime}\right)$ for the sake of simplicity. In Kriging, the limit state function $g\left(\mathbf{X}, \mathbf{Y}^{\prime}, t^{\prime}\right)$ is generated from a surrogate model as

$$
g_{K}(w)=\mathbf{f}^{\mathbf{T}}(w) \boldsymbol{\alpha}+S(w)
$$

where $g_{K}(w)$ is an approximation of the true limit state function $g(w)$ by the surrogate model, $w$ is a realization of the random input $\mathbf{W}, \mathbf{f}^{\mathbf{T}}(w)$ is a set of basis functions, $\boldsymbol{\alpha}$ is a regression coefficient vector, and $S(w)$ is a Gaussian random process with zero mean and a covariance matrix. As there is no prior information about the global trend of the limit state function $g\left(\mathbf{X}, \mathbf{Y}^{\prime}\right.$, $\left.t^{\prime}\right)$, a constant mean $\mu$ is assumed in the surrogate model to replace the term $\mathbf{f}^{\mathbf{T}}(w) \boldsymbol{\alpha}$.

For the stochastic process $S(w)$, the covariance function between two input points $w_{i}$ and $w_{j}$ is expressed as

$$
\operatorname{Cov}_{(i, j)}=\sigma^{2} \mathbf{R}_{(i, j)}
$$

where $\mathbf{R}$ is the correlation matrix. The $(i, j)$ entry of $\mathbf{R}$ is described as

$$
\mathbf{R}_{(i, j)}=\exp \left[-\sum_{u=1}^{k} a_{u}\left|w_{i, u}-w_{j, u}\right|^{b_{u}}\right],
$$


where $k=(n+m+1)$ is the number of variables in $\mathbf{W}, a_{u}$ and $b_{u}(u=1, . ., k)$ are hyper parameters of the Kriging model. With $p$ data points $\left(\mathbf{W}_{p}, \boldsymbol{G}_{\boldsymbol{p}}\right)$, the log likelihood function is expressed as

$$
\log =-\frac{1}{2}\left[p \ln (2 \pi)+p \ln \sigma^{2}+\ln |\mathbf{R}|+\frac{1}{2 \sigma^{2}}\left(\mathbf{G}_{\mathbf{p}}-\mathbf{A} \mu\right)^{T} \mathbf{R}^{-1}\left(\mathbf{G}_{\mathbf{p}}-\mathbf{A} \mu\right)\right]
$$

where $\mathbf{A}$ is an $p \times 1$ unit vector. All hyper parameters of Kriging model are obtained by maximizing the likelihood function in Eq. (16). The Kriging model is capable of predicting the limit state value for any input point $w^{\prime}$ as

$$
g_{K}\left(w^{\prime}\right)=\mu+\mathbf{r}^{T} \mathbf{R}^{-1}\left(\mathbf{G}_{\mathbf{P}}-\mathbf{A} \mu\right)
$$

where $\mathbf{r}$ is the correlation vector between $w^{\prime}$ and the samples $\mathbf{W}_{\mathbf{p}}$. Due to a lack of data, the mean square error $e\left(w^{\prime}\right)$ is estimated by

$$
\hat{e}\left(w^{\prime}\right)=\sigma^{2}\left[1-\mathbf{r}^{T} \mathbf{R}^{-1} \mathbf{r}+\frac{\left(1-\mathbf{A}^{T} \mathbf{R}^{-1} \mathbf{r}\right)^{2}}{\mathbf{A}^{T} \mathbf{R}^{-1} \mathbf{A}}\right]
$$

\subsection{Adaptive Sampling Approach}

In order to solve the reliability problem defined by the instantaneous reliability model, the Kriging surrogate model is built to predict the limit state function $g\left(\mathbf{X}, \mathbf{Y}^{\prime}, t^{\prime}\right)$, which thus enables the approximation of the time-invariant probability of failure using MCS. To do so, a large number of random samples are generated according to the randomness of parameters $\mathbf{X}$, Y'and $t$ ', and then evaluated based on the surrogate model for responses. Let $\Omega=\left\{\mathbf{w}, \mid g_{K}(\mathbf{w})>0\right\}$ denote failure area, the time-invariant probability of failure is expressed as

$$
P_{f}=\operatorname{Pr}\left(g_{K}(\mathbf{W}) \in \Omega\right)=\int_{R^{k}} I(w) f_{w}(w) d w=\mathrm{E}[I(w)]
$$

where $\operatorname{Pr}($.$) represents a probability measure; f_{w}(w)$ is the probability density function of random input parameters $\mathbf{W}$; E[.] denotes the expectation operator; $R^{k}$ is a $k$-dimensional real number space; $I(w)$ is an indicator function defined as 


$$
I(w)=\left\{\begin{array}{ll}
1, & g_{K}(w)>0 \\
0, & \text { otherwise }
\end{array},\right.
$$

where $g_{K}(w)$ is the prediction of the limit state value for input $\mathrm{w}$. Thus, the time-invariant probability of failure is derived as

$$
P_{f} \approx \frac{1}{N} \sum_{i=1}^{N} I\left(w_{i}\right)
$$

where $N$ is the total number of sample points evaluated in MCS.

Due to a limited amount of data used in constructing Kriging models, a major issue of Kriging models lies in the difficulty of quantitatively measuring the surrogate model fidelity for reliability computations, as well as the development of advanced adaptive sampling algorithms for surrogate model updating. In this work, the maximum confidence enhancement (MCE) based sequential sample scheme [9] is employed to enhance the fidelity of Kriging models for predicting the instantaneous failure surface. In MCE, the probability of correct classification for the $i^{\text {th }}$ sample point $w_{i}(1 \leq i \leq N)$ is derived as

$$
\operatorname{Pr}_{c}\left(w_{i}\right)=\Phi\left(\frac{\left|g_{K}\left(w_{i}\right)\right|}{\sqrt{\hat{e}\left(w_{i}\right)}}\right),
$$

where |.| is the absolute operator. Thus a quantitative measure, the cumulative confidence level of reliability approximations for Kriging models [9] is obtained as

$$
C C L=\mathrm{E}\left[\mathbf{P r}_{\mathbf{c}}\right]=\frac{1}{N} \sum_{i=1}^{N} \operatorname{Pr}_{c}\left(w_{i}\right)
$$

It is worth noting that the measure $C C L$ is a positive value within $(0.5,1]$. Clearly, $C C L$ indicates the confidence of reliability approximations and decreases with the fidelity of surrogate models. It is also worth noting that $C C L$ does not measure the global fidelity of surrogate model, instead, it indicates the accuracy level on critical local areas, the instantaneous failure surface, which is 
critical for reliability predictions.

In order to improve the efficacy of Kriging models in predicting the instantaneous failure surface, an adaptive sampling scheme developed based on the maximum confidence enhancement (MCE) method is utilized to iteratively update the Kriging models. In MCE, the most useful sample point should be selected within the MCS sample pool $\mathbf{W}=\left[w_{1}, . ., w_{N}\right]$ by calculating an importance measure, which is expressed as

$$
\psi\left(w_{i}\right)=\left(1-\operatorname{Pr}_{c}\left(w_{i}\right)\right) \times f_{w}\left(w_{i}\right) \times \sqrt{\hat{e}\left(w_{i}\right)},
$$

where $\operatorname{Pr}_{c}\left(w_{i}\right)$ represents the probability of correct classification; $f_{w}\left(w_{i}\right)$ is the probability density value at $w_{i} ; \hat{e}\left(w_{i}\right)$ is the estimated mean square error of the Kriging model prediction. Then, the most useful sample point $w^{\prime}$ is identified by maximizing $\psi($.$) as$

$$
w^{\prime}=\underset{i}{\arg \max } \psi\left(w_{i}\right), i=1, \ldots, N .
$$

The true performance of dynamic systems is thus evaluated for the identified point $w^{\prime}$, leading to a new Kriging surrogate model by incorporating the new observations. The updating mechanism is performed repeatedly till the cumulative conformance level satisfies a predefined confidence target. Normally, a greater confidence target will result in a more accurate reliability approximation, which may require more computational efforts. It is therefore a tradeoff in setting the confidence target between accuracy and efficiency. It is recommended that a greater confidence level target should be used for the cases with a smaller probability of failure.

\subsection{Time-Variant Reliability Approximation}

As a simulation-based method, direct MCS for time-variant reliability assessment involves generating a large number of random samples for variables $\mathbf{X}$ and realizations of random processes $\mathbf{Y}(t)$, and evaluating time-dependent responses for counting the number of failure events during a specified time period. To reduce computational efforts, the Kriging model $g_{K}(w)$ 
is utilized to predict the true response and thus identify instantaneous failure events at any time instant.

In time-variant reliability approximation, the first step is to generate random realizations of random inputs $\mathbf{X}$ and $\mathbf{Y}(t)$. Another advantage of the eSPT method lies in that there is no assumption about $\mathbf{Y}(t)$ and thus both Gaussian processes and non-Gaussian processes can be handled in the time-variant reliability analysis. Despite eSPT being capable of addressing general stochastic processes, numerical simulation of Gaussian processes is introduced in this work to generate random realizations. More references about numerical simulations of non-Gaussian random processes can be found in [32-34]. A Gaussian process $Y(t)$ can be prescribed by a mean function $\mu_{\mathrm{Y}}(t)$, standard deviation function $\sigma_{\mathrm{Y}}(t)$, and autocorrelation function $\rho_{\mathrm{Y}}\left(t_{i}, t_{j}\right)$. With a discretized time interval $[0, T]$ by $s$ time nodes, the covariance between two time instants $t_{i}$ and $t_{j}$ is calculated by $\operatorname{Cov}\left(t_{i}, t_{j}\right)=\sigma_{\mathrm{Y}}\left(t_{i}\right) \sigma_{\mathrm{Y}}\left(t_{j}\right) \rho_{\mathrm{Y}}\left(t_{i}, t_{j}\right)$. The covariance matrix $\boldsymbol{\Sigma}$ is formed as

$$
\Sigma=\left(\begin{array}{cccc}
\operatorname{Cov}\left(t_{1}, t_{1}\right) & \operatorname{Cov}\left(t_{1}, t_{2}\right) & \ldots & \operatorname{Cov}\left(t_{1}, t_{s}\right) \\
\operatorname{Cov}\left(t_{2}, t_{1}\right) & \operatorname{Cov}\left(t_{2}, t_{2}\right) & \cdots & \operatorname{Cov}\left(t_{2}, t_{s}\right) \\
\vdots & \vdots & \ddots & \vdots \\
\operatorname{Cov}\left(t_{s}, t_{1}\right) & \operatorname{Cov}\left(t_{s}, t_{1}\right) & \cdots & \operatorname{Cov}\left(t_{s}, t_{s}\right)
\end{array}\right),
$$

By performing Eigen decomposition of covariance matrix, the Gaussian process $\mathrm{Y}(t)$ is reconstructed as

$$
, Y(t) \quad{ }_{Y}(t)+{ }_{i=1}^{p} \sqrt{I_{i}} \times_{i}(t) \bigcup_{i}
$$

where $\boldsymbol{\Phi}=\left[\Phi_{1}, \Phi_{2}, \ldots, \Phi_{\mathrm{s}}\right]$ is the eigenvectors of the matrix $\boldsymbol{\Sigma}$, $\mathbf{I}=\operatorname{diag}\left[\mathrm{I}_{1}, \mathrm{I}_{2}, \ldots, \mathrm{I}_{\mathrm{s}}\right]$ is a diagonal matrix that contains the corresponding eigenvalues, $p$ is the number of dominated eigenfunction, $\mathbf{U}=\left[U_{1}, \ldots, U_{p}\right]$ are a set of uncorrelated standard normal distributed random variables.

Let $Y_{i}=\left[y_{1}, \ldots, y_{i}, \ldots, y_{s}\right]$ represent the $i^{\text {th }}$ of the total $N$ realizations of the stochastic process 
$\mathrm{Y}(t)$ in MCS where $y_{j}$ is the value of the stochastic process at the $j^{\text {th }}$ time instant. The

performance at the $j^{\text {th }}$ time instant is thus predicted as $g_{\mathrm{K}}\left(x_{i}, y_{i}, t_{i}\right)$ by the Kriging surrogate model, and an instantaneous failure is identified if $g_{\mathrm{K}}\left(x_{i}, y_{i}, t_{i}\right)>0$. Similarly, the time-dependent limit state function is evaluated by the Kriging model, and a process failure occurs in time-variant reliability analysis if

$$
\max _{1 \leq j \leq s} g_{K}\left(x_{i}, y_{j}, t_{j}\right)>0 .
$$

The probability of failure for time interval $[0, T]$ is thus calculated by

$$
P_{f}(0, T) \approx N_{f} / N,
$$

where $N_{f}$ indicates the number of process failure events out of the total $N$ random trajectories.

\section{CASE STUDIES}

In this section, two case studies, a mathematical example and an engineering application, are used to illustrate the performance of the eSPT approach.

\subsection{Case Study I: A Math Example}

A benchmark problem is solved to demonstrate the performance of the eSPT method for time-variant reliability assessment. The time-dependent limit state function is given by

$$
g(\mathbf{X}, \mathbf{Y}(t), t)=20-X_{1}^{2} X_{2}+5 X_{1}(1+Y(t)) t-\left(X_{2}+1\right) t^{2},
$$

where $t$ is the time parameter varying within $[0,1] ; \mathbf{X}=\left[X_{1}, X_{2}\right]$ are normally distributed random variables with 3.5 mean and 0.25 standard deviation, $Y(t)$ is a Gaussian process with zero mean and unit variance. The correlation of the random process $Y(t)$ is defined as

$$
\rho\left(t_{1}, t_{2}\right)=\exp \left(-\left(t_{2}-t_{1}\right)^{2}\right) .
$$


As a reference, a direct MCS is carried out to calculate the time-variant reliability with $10^{6}$ random sample points, whose function values are evaluated directly by Eq. (30). With the direct MCS, the random process $Y(t)$ is discretized into 200 time intervals evenly. By performing Eigen analysis of the covariance matrix, the random process $Y(t)$ is reconstructed by spectral decomposition. In this way, an accurate reliability history over time is obtained by the direct MCS and used as a reference solution for comparison.

Next, the proposed eSPT method is compared with the out-crossing rate based method in terms of efficacy and accuracy for this example. With eSPT, an instantaneous reliability model is constructed as $g\left(\mathbf{X}, \mathbf{Y}^{\prime}, t^{\prime}\right)$ where $t^{\prime}$ is a uniformly distributed random variable at interval $[0,1]$. As $\mathbf{Y}(t)$ is a Gaussian process with zero mean and unit variance, $\mathbf{Y}^{\prime}$ is a random variable which follows a standard normal distribution. With a space domain defined by the probabilistic attributes of inputs $\mathbf{W}=\left[X_{1}, X_{2}, \mathbf{Y}^{\prime}, t^{\prime}\right], 40$ points are randomly generated using the Latin hypercube sampling technique and evaluated for the function output. An initial low fidelity Kriging surrogate model is first constructed to approximate the instantaneous failure surface. Using MCS, the instantaneous reliability is approximated as $0.8^{*} 10^{-3}$, which is much smaller than the true value 0.1021 due to the low fidelity of the failure surface at this stage. The cumulative confidence level is 0.513 , indicating that the approximation of instantaneous failure surface by the Kriging model is not accurate. The instantaneous failure surface by fixing $\mathbf{Y}^{\prime}=0$ and $t^{\prime}=0.5$ is illustrated in Figure 5. Clearly, the failure surface predicted by the initial low fidelity Kriging model lacks the capability of predicting instantaneous failures due to lack of data. 


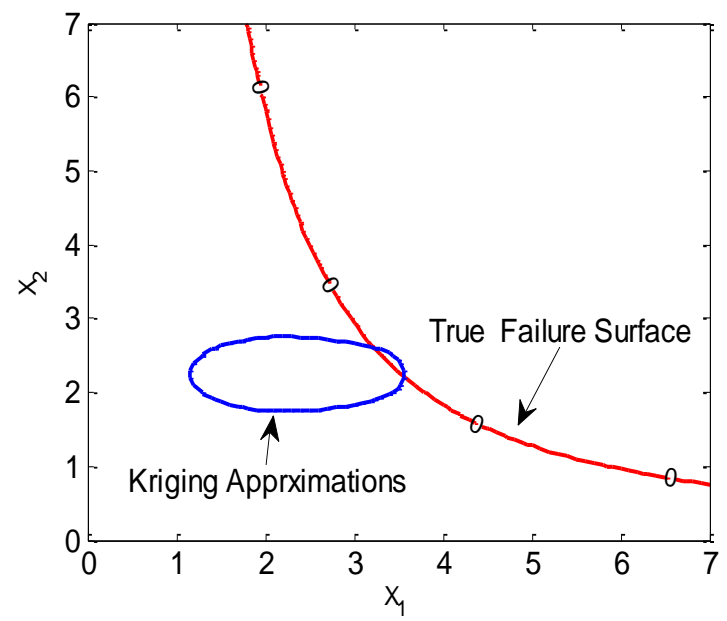

Figure 5: Low-Fidelity of the Initial Kriging Model

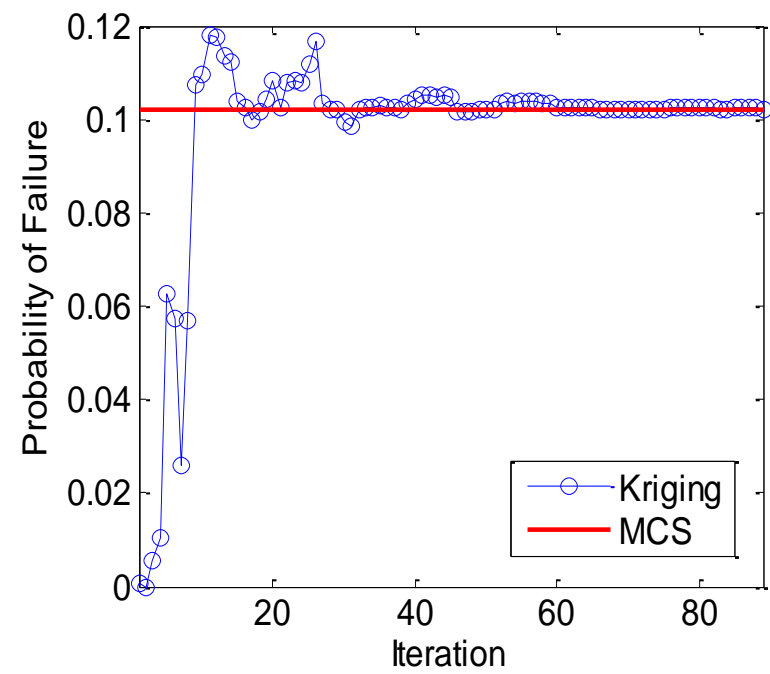

Figure 6: Iterative Surrogate Updating for Reliability Analysis

The adaptive sampling scheme introduced in Section 3.4 is then triggered to pinpoint the most useful sample point for updating the Kriging model. As shown in Figures 6 and 7, with 89 iterations the cumulative confidence level reaches 0.999 and a probability of failure is obtained as 0.1022 , which converges to the true value while the confidence level increases. With the updated Kriging model, the instantaneous failure surface is accurately obtained for future analysis in time-variant reliability assessment. Figure 8 demonstrates the approximated 
instantaneous failure surface using the updated Kriging model by fixing $\mathbf{Y}^{\prime}=0$ and $t^{\prime}=0.5$. The fidelity of Kriging models at important region in the vicinity of true failure surface is of critical importance in approximating time-variant reliability. In this work, the maximum confidence enhancement (MCE) based adaptive sampling scheme is integrated to efficiently identify the true failure surface and enhance the fidelity of Kriging models.

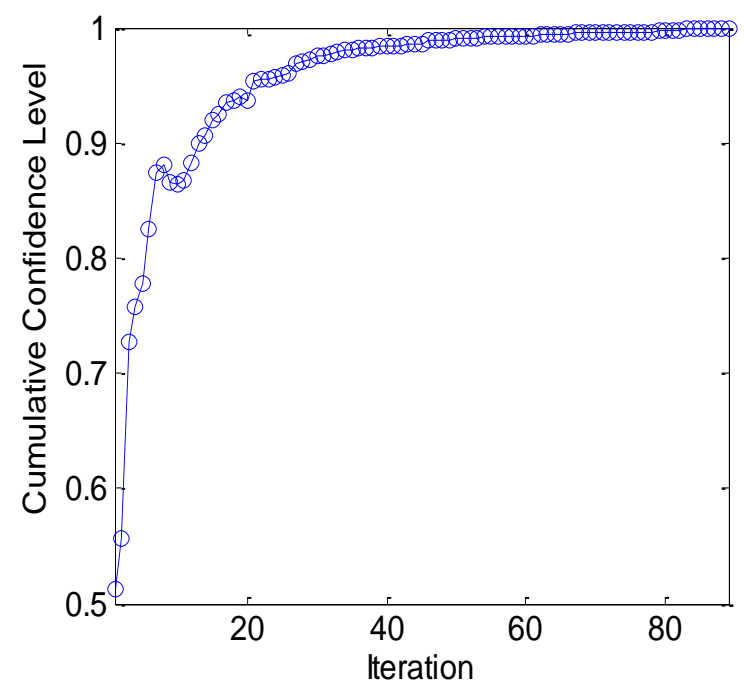

Figure 7: Cumulative Confidence Level during Updating Process

The extreme value distribution of limit state function within a time interval $[0,0.5]$ is illustrated in Figure 9. As eSPT is not designed to obtain an accurate approximation of the extreme value distribution, the shape of the probability density function of the extreme value approximated by the eSPT is quite different from MCS results. Nevertheless, the right tail of the probability density curve is exactly the same with the one obtained by MCS, leading to an accurate approximation of time-variant reliability. In addition, vast computational efforts can be saved for searching the extreme performances over time scale, which is usually required in existing extreme value-based time-variant reliability approaches.

In addition, the out-crossing rate based approach [13] is employed to solve the same benchmark problem to demonstrate the efficiency and accuracy of eSPT. With the out-crossing 
rate based method, time is discretized by 20 time intervals evenly and thus FORM is employed to search the MPPs for each time instant. The results from eSPT, out-crossing rate-based approach and direct MCS are shown in Figure 10 and Table 1. Especially, eSPT only requires 129 performance evaluations of the time-dependent limit state function including 40 evaluations for constructing the initial Kriging model and another 89 evaluations for updating the model. As widely applied analytical approaches, the out-crossing rate based approach requires 1116 evaluations of the limit state function, which indicates that the proposed approach is superior in terms of both efficiency and accuracy.

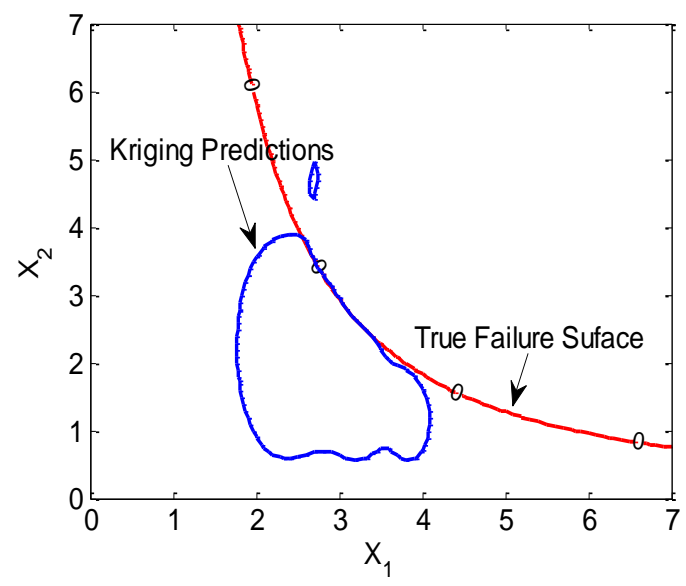

Figure 8: High-Fidelity of the Updated Kriging Model

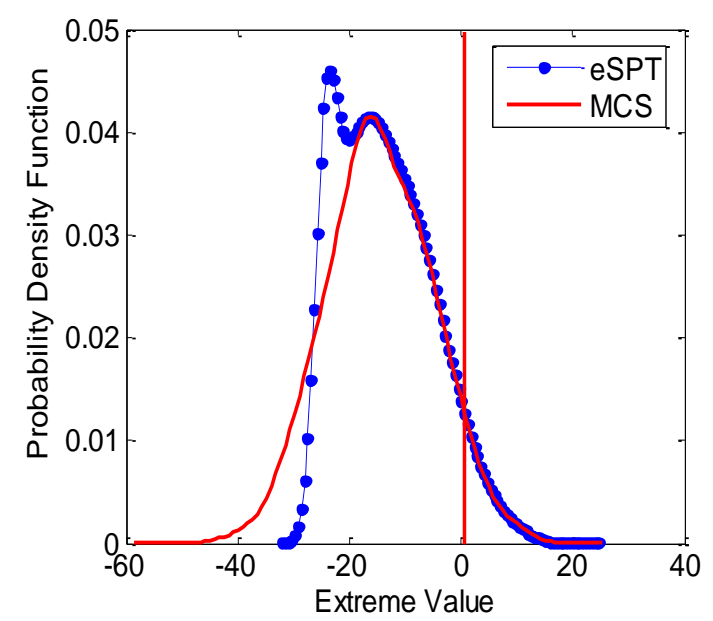

Figure 9: Extreme Value Distributions for time interval [0, 0.5] 


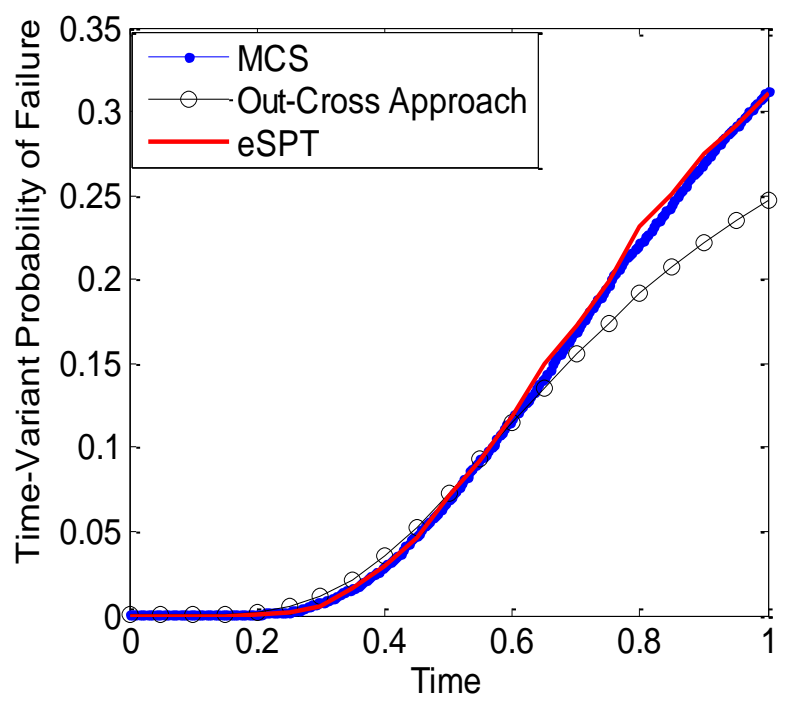

Figure 10: Time-Variant Probability of Failure

Table 1 Time-Variant Probability of Failure

\begin{tabular}{cccc}
\hline \hline Time Interval & $\begin{array}{c}\text { 95\% Confidence } \\
\text { MCS }\end{array}$ & eSPT & 'Out-Crossing Rate Method \\
\hline$[0,0.4]$ & {$[0.0293,0.0299]$} & 0.0278 & 0.03511 \\
{$[0,0.6]$} & {$[0.1184,0.1196]$} & 0.1161 & 0.11458 \\
{$[0,0.8]$} & {$[0.2221,0.2237]$} & 0.2152 & 0.19162 \\
{$[0,1.0]$} & {$[0.2994,0.3012]$} & 0.2986 & 0.24701 \\
$\#$ of Fun & $10^{6}$ & $40+89$ & 1116 \\
\hline \hline
\end{tabular}

\subsection{Case Study II: Hydrokinetic Turbine Blade}

As a renewable energy device, hydrokinetic turbine converts the kinetic energy of flowing water into electrical energy. The river flow load is time-dependent and is often characterized using a random process. Achieving a high life-cycle reliability is of critical importance in the early design stage of hydrokinetic turbine blade to reduce the operational maintenance cost. In this study, the proposed instantaneous failure approach is utilized to assess the time-variant reliability of a given design of turbine blades [35].

To simulate the time-dependent river flow, the monthly river velocity is characterized by a 
random process $\mathbf{Y}(t)$ with mean $\mu_{\mathrm{y}}(t)$, standard deviation $\sigma_{\mathrm{y}}(t)$, and autocorrelation function $\rho_{\mathrm{y}}\left(t_{1}, t_{2}\right)$ given by

$$
\begin{gathered}
\mu_{y}(t)=\sum_{i=1}^{4} a_{i}^{m} \sin \left(b_{i}^{m} t+c_{i}^{m}\right), \\
\sigma_{y}(t)=\sum_{j=1}^{4} a_{i}^{s} \exp \left\{-\left[\left(t-b_{j}^{s}\right) / c_{j}^{s}\right]\right\}, \text { and } \\
\rho_{y}\left(t_{1}, t_{2}\right)=\cos \left(2 \pi\left(t_{2}-t_{1}\right)\right),
\end{gathered}
$$

where the constants $a, b, c$ for Equations. (33) and (34) are

$$
\begin{gathered}
a_{1}^{m}=3.815, a_{2}^{m}=2.528, a_{3}^{m}=1.176, a_{4}^{m}=-0.07856 \\
b_{1}^{m}=0.2895, b_{2}^{m}=0.5887, b_{3}^{m}=0.7619, b_{4}^{m}=2.183 \\
c_{1}^{m}=-0.2668, c_{2}^{m}=0.9651, c_{3}^{m}=3.116, c_{4}^{m}=-3.161 \\
a_{1}^{s}=0.7382, a_{2}^{s}=1.013, a_{3}^{s}=1.875, a_{4}^{s}=1.283 \\
b_{1}^{s}=6.456, b_{2}^{s}=4.075, b_{3}^{s}=9.913, b_{4}^{s}=1.035 \\
c_{1}^{s}=0.9193, c_{2}^{s}=1.561, c_{3}^{s}=6.959, c_{4}^{s}=2.237
\end{gathered}
$$

Under the stochastic water load, the bending moment at the root of the hydrokinetic turbine blade shown in Figure 11 is expressed as

$$
M_{b}=\frac{1}{2} \rho \mathbf{Y}^{2}(t) C_{m}
$$

where $\rho=10^{3} \mathrm{~kg} / \mathrm{m}^{3}$ is the water density, and $C_{m}=0.3422$ represents the coefficient of moment. The time-dependent limit state function of hydrokinetic turbine blades is thus defined by

$$
g\left(d_{1}, d_{2}, d_{3}, A_{l}, \mathbf{Y}(t)\right)=\frac{d_{1} M_{b}}{E I_{a}}-A_{l}
$$

where $A_{l}$ is the allowable material stain, $M_{b}$ is the flapwise bending moment, and $E=14 \mathrm{GPa}$ is the Young's modulus. The time period is set as 12 months. The area moment of inertia at the root of the blade is derived as 


$$
I_{a}=\frac{2}{3} d_{3}\left(d_{1}^{3}-d_{2}^{3}\right) .
$$

The detailed information of the random parameters $d_{1}, d_{2}, d_{3}, A_{l}$ and the random process $\mathrm{v}(\mathrm{t})$ are listed in Table 2.

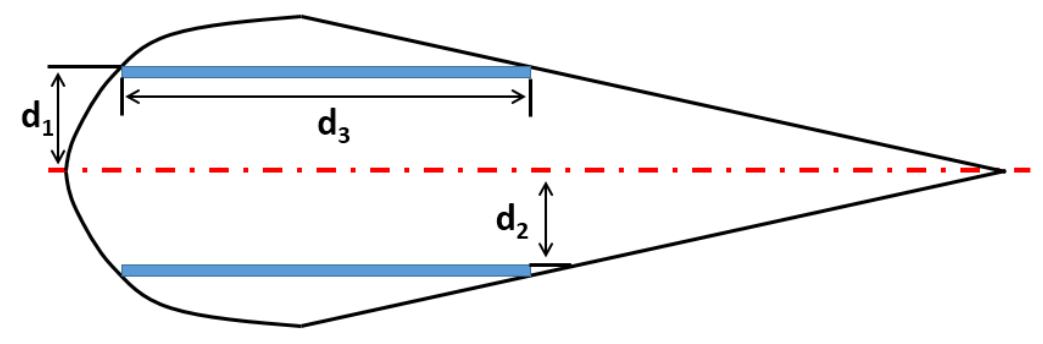

Figure 11: Hydrokinetic Tubing Blade

Table 2: Random Variables for Hydrokinetic Turbine Blade

\begin{tabular}{ccccc}
\hline \hline Variables & Distribution & Mean $(\mathrm{mm})$ & Std Dev. $(\mathrm{mm})$ & Auto Cor. \\
\hline$d_{1}$ & Normal & 0.22 & 0.00220 & N/A \\
$d_{2}$ & Normal & 0.025 & 0.00025 & N/A \\
$d_{3}$ & Normal & 0.019 & 0.00019 & N/A \\
$A_{l}$ & Normal & 0.015 & 0.00015 & N/A \\
$\mathbf{Y}(t)$ & GP & $\mu_{\mathrm{y}}(\mathrm{t})$ & $\sigma_{\mathrm{y}}(\mathrm{t})$ & $\rho_{\mathrm{y}}(\mathrm{t})$ \\
\hline \hline
\end{tabular}

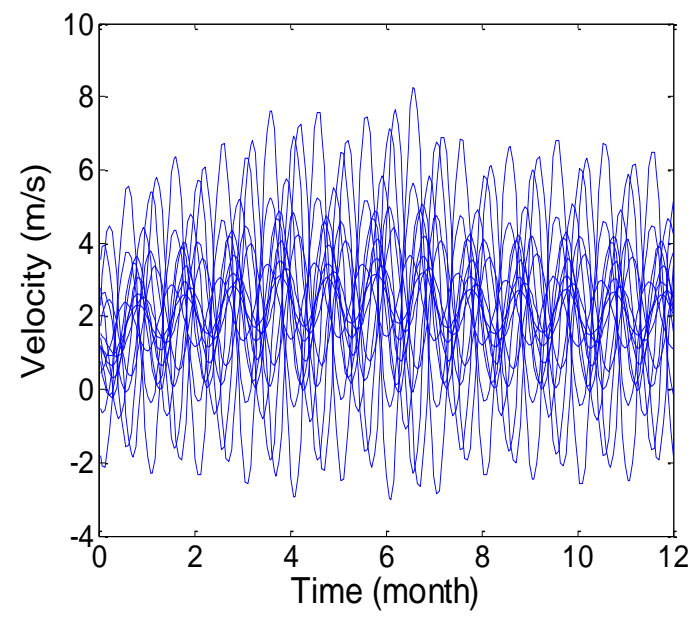

Figure 12: Realizations of the Random Process $\mathbf{Y}(t)$ 
To obtain an accurate instantaneous failure surface, an instantaneous reliability model is constructed with a translation of the input random process $\mathbf{Y}(t)$. Figure 12 demonstrates the realizations of the random process $\mathbf{Y}(t)$. The probability density function (PDF) of the translated random variable $\mathbf{Y}^{\prime}$ is obtained by the average of the PDF of the random process $\mathbf{Y}(t)$ over time period $[0,12]$. Figure 13 shows that the approximated probability density functions of $\mathbf{Y}^{\prime}$ and the random process $\mathbf{Y}(t)$ with $t=[0,6,12]$ respectively. As the time parameter is implicitly involved in the time-dependent limit state function simulation, the instantaneous reliability model is formulated as $g\left(d_{1}, d_{2}, d_{3}, A_{l}, \mathbf{Y}^{\prime}\right)$ without the $t$ ' parameters. This is because the reliability degradation effect can be fully captured by the stochastic process parameter $\mathbf{Y}(t)$. Therefore, within a five-dimensional input space, 50 random samples are generated randomly by the Latin hypercube sampling technique and evaluated for the responses. An initial low-fidelity Kriging model is built to predict the response of $g\left(d_{1}, d_{2}, d_{3}, A_{l}, \mathbf{Y}^{\prime}\right)$. In solving the formulated instantaneous reliability problem, the Kriging model is iteratively updated to enhance the fidelity of the Kriging model for predicting the instantaneous failure surface. Figures 14 and 15 demonstrate the convergence process of reliability approximations and the updating process of cumulative confidence levels.

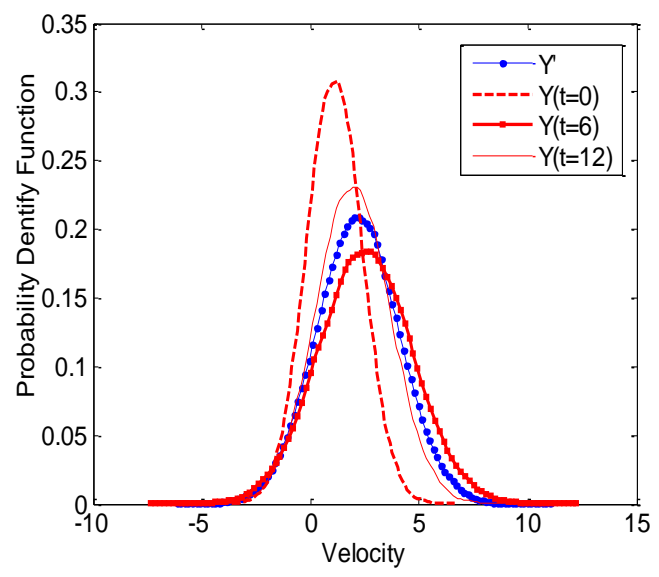

Figure 13: Translation of the Random Process $\mathbf{Y}(t)$ 


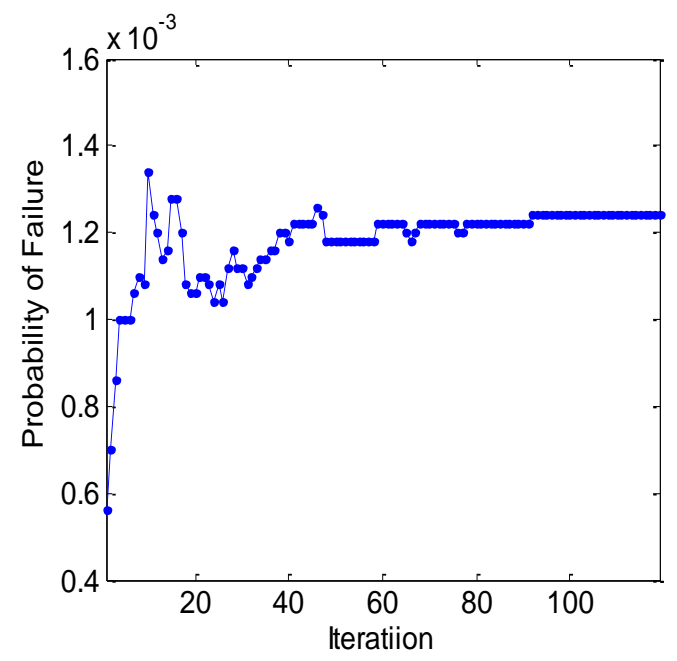

Figure 14: Probability of Failure

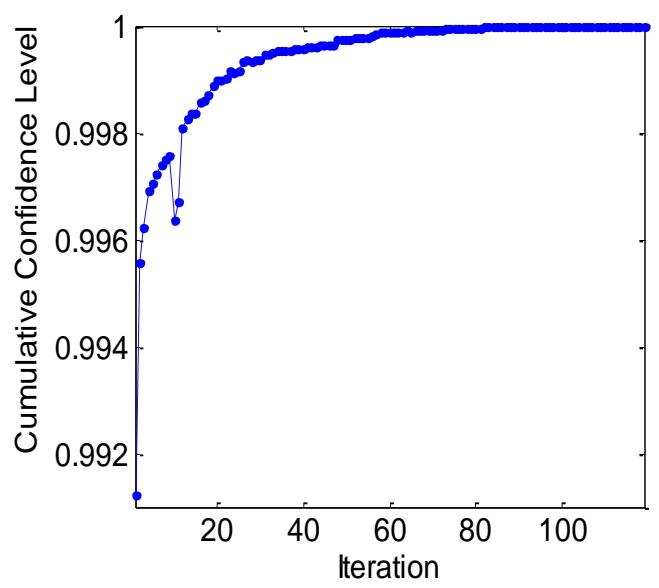

Figure 15: Cumulative Confidence Level

After 119 iterations, 119 critical sample points are identified and evaluated to update the Kriging model, resulting in an accurate surrogate model in predicting the instantaneous failure surface. With the accurate surrogate model, the time-variant reliability is readily approximated by MCS. To check the accuracy of the proposed approach, a direct MCS with $10^{6}$ samples is also employed for solving the hydrokinetic turbine blade reliability problem. The results from both 
methods are demonstrated in Figure 16, indicating that the eSPT approach is able to assess the time-variant reliability accurately while requiring much less simulation runs.

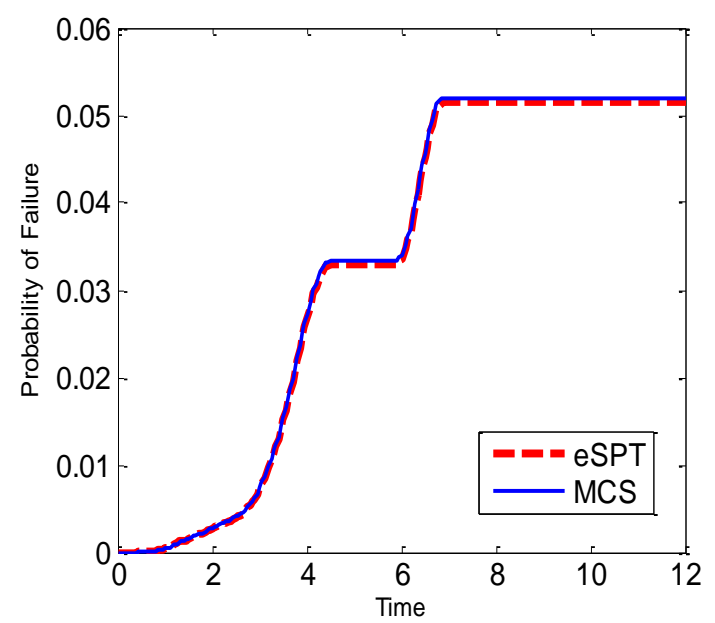

Figure 16: Time-variant Probability of Failure

\section{CONCLUSION}

This paper presented an equivalent stochastic process transformation approach for costeffective prediction of reliability deterioration over the life cycle of engineered systems with the consideration of both random variables and random process parameters. A novel concept, referred to as instantaneous failure surface, is introduced to cover all potential failure events that may occur within a certain period of time. To obtain the instantaneous failure surface, a timeinvariant reliability problem is formulated by translating the random processes and time parameter into random variables. Kriging techniques are utilized to construct a surrogate model to predict the instantaneous failure surface, and the surrogate model is updated effectively using the maximum confidence enhancement sequential sampling scheme. The time-variant reliability of dynamic systems is evaluated by the Monte Carlo simulations based upon the updated highfidelity Kriging model. The proposed approach is used to solve a math benchmark problem and 
to evaluate the time-variant reliability of a hydrokinetic turbine blade. The results illustrate that the proposed eSPT method is superior to existing approaches in terms of both accuracy and efficiency. For future work, the eSPT approach will be extended for time-dependent reliabilitybased design optimization.

\section{ACKNOWLEDGEMENT}

This work was performed under the financial assistance award 70NANB14H012 from U.S. Department of Commerce, National Institute of Standards and Technology as part of the Center for Hierarchical Materials Design (CHiMaD), and also partially supported through a subcontract from the Ford Motor Company with funding from the U.S. Department of Energy's Office of Energy Efficiency and Renewable Energy (EERE), under award number DEEE0006867.

\section{REFERENCES}

1. Youn, Byeng D., Choi, K. K., and Du, L., 2005, “Adaptive Probability Analysis Using An Enhanced Hybrid Mean Value (HMV+) Method," Journal of Structural and Multidisciplinary Optimization, Vol. 29, No. 2, pp.134-148.

2. Youn, B.D., Choi, K. K., and Du, L., 2005, "Enriched Performance Measure Approach (PMA+) for Reliability-Based Design Optimization,” AIAA Journal, Vol. 43, No. 4, pp. 874884.

3. Rahman, S. and Xu, H., 2004, "A Univariate Dimension-Reduction Method for MultiDimensional Integration in Stochastic Mechanics," Probabilistic Engineering Mechanics, Vol. 19, pp. 393-408. 
4. Youn, B. D., Xi Z., and Wang, P., 2008, "Eigenvector Dimension-Reduction (EDR) Method for Sensitivity-Free Uncertainty Quantification,” Struct. Multidiscipl. Optim., Vol. 37, No.1, pp13-28.

5. Xu, H., and Rahman, S., 2005, "Decomposition methods for structural reliability analysis," Probabilistic Engineering Mechanics, 20(3), pp. 239-250.

6. Hu, C, and Youn, B.D., 2011, "Adaptive-sparse polynomial chaos expansion for reliability analysis and design of complex engineering systems." Structural and Multidisciplinary Optimization, 43, no. 3, 419-442.

7. Crestaux, Thierry, Olivier Le Ma^^tre, and Jean-Marc Martinez. "Polynomial chaos expansion for sensitivity analysis." Reliability Engineering \& System Safety 94.7 (2009): 1161-1172.

8. Sudret, Bruno., 2008, "Global sensitivity analysis using polynomial chaos expansions." Reliability Engineering \& System Safety, 93, no. 7, 964-979.

9. Wang, Z., and Wang, P., 2013, “A Maximum Confidence Enhancement Based Sequential Sampling Scheme for Simulation-Based Design”, J. Mech. Des., In Press.

10. Dey, Animesh, and Sankaran Mahadevan. "Ductile structural system reliability analysis using adaptive importance sampling." Structural Safety 20.2 (1998): 137-154.

11. Kaymaz, Irfan. "Application of kriging method to structural reliability problems." Structural Safety 27.2 (2005): 133-151.

12. Lutes, L. D., and Sarkani, S., 2009, "Reliability Analysis of Systems Subject to First-Passage Failure," NASA Technical Report No. NASA/CR-2009-215782.

13. Andrieu-Renaud, C., Sudret, B., and Lemaire, M., 2004, "The PHI2 Method: A Way to Compute Time-Variant Reliability," Reliab. Eng. Syst. Saf., 84(1), pp.75-86. 
14. Hu, Z. and Du, X., 2013, "Time-Dependent Reliability Analysis with Joint Up-crossing Rates," Structural and Multidisciplinary Optimization, 2013, DOI: 10.1007/s00158-0130937-2.

15. Rice, S. O., 1944, "Mathematical Analysis of Random Noise,” Bell Syst. Tech. J., 23, pp. $282-332$.

16. Breitung, Karl. "Asymptotic crossing rates for stationary Gaussian vector processes." Stochastic processes and their applications 29.2 (1988): 195-207.

17. Engelund, S., R. Rackwitz, and C. Lange. "Approximations of first-passage times for differentiable processes based on higher-order threshold crossings." Probabilistic Engineering Mechanics 10.1 (1995): 53-60.

18. Hu, Zhen, and Xiaoping Du. "First order reliability method for time-variant problems using series expansions." Structural and Multidisciplinary Optimization 51.1 (2015): 1-21.

19. Wang, Zequn, and Pingfeng Wang. "A new approach for reliability analysis with timevariant performance characteristics." Reliability Engineering \& System Safety 115 (2013): $70-81$.

20. Chen, J.-B., and Li, J., 2007, “The Extreme Value Distribution and Dynamic Reliability Analysis of Nonlinear Structures with Uncertain Parameters,” Struct. Safety, 29(2), pp. 77-93.

21. Wang, Z., and Wang, P., 2012, “A Nested Extreme Response Surface Approach for TimeDependent Reliability-Based Design Optimization,” J. Mech. Des. 134, 121007

22. Li, J., and Mourelatos, Z. P., 2009, "Time-Dependent Reliability Estimation for Dynamic Problems Using a Niching Genetic Algorithm," Journal of Mechanical Design, 131(7), p. 071009.

23. Mourelatos ZP, Majcher M, Pandey V, Baseski I. Time-Dependent Reliability Analysis 
Using the Total Probability Theorem. ASME. J. Mech. Des. 2015;137(3):031405-031405-8

24. Gaspar, B., Teixeira, A.P. \& Guedes Soares, C. (2014). Assessment of the efficiency of Kriging surrogate models for structural reliability analysis. Probabilistic Engineering Mechanics, 37, 24-34.

25. Chojaczyk, A.A., Teixeira, A.P., Neves, L.C., Cardoso, J.B. \& Guedes Soares, C. (2015). Review and application of Artificial Neural Networks models in reliability analysis of steel structures. Structural Safety, 52, 78-89.

26. Bucher, C. \& Most, T. (2008). A comparison of approximate response functions in structural reliability analysis. Probabilistic Engineering Mechanics, 23, 154-163.

27. Sudret, B. (2012). Meta-models for structural reliability and uncertainty quantification. In Fifth Asian-Pacific Symposium on Structural Reliability and its Applications (5APSSRA), Phoon, K.K., Beer, M., Quek, S.T. \& Pang, S.D. (eds.), 53-76, Singapore.

28. Dubourg, Vincent, B. Sudret, and F. Deheeger. "Metamodel-based importance sampling for structural reliability analysis." Probabilistic Engineering Mechanics 33 (2013): 47-57.

29. Gaspar, B., A. P. Teixeira, and C. Guedes Soares. "Assessment of the efficiency of Kriging surrogate models for structural reliability analysis." Probabilistic Engineering Mechanics 37 (2014): 24-34.

30. Zhang, Leigang, Zhenzhou Lu, and Pan Wang. "Efficient structural reliability analysis method based on advanced Kriging model." Applied Mathematical Modelling 39.2 (2015): 781-793.

31. Zhao, W., et al. "A moving kriging interpolation response surface method for structural reliability analysis." Comput. Model. Eng. Sci 93.6 (2013): 469-488.

32. Deodatis, G., \& Micaletti, R. C. (2001). Simulation of highly skewed non-Gaussian 
stochastic processes. Journal of engineering mechanics, 127(12), 1284-1295.

33. Sakamoto, Shigehiro, and Roger Ghanem. "Polynomial chaos decomposition for the simulation of non-Gaussian nonstationary stochastic processes." Journal of engineering mechanics 128.2 (2002): 190-201.

34. Sakamoto, Shigehiro, and Roger Ghanem. "Simulation of multi-dimensional non-Gaussian non-stationary random fields." Probabilistic Engineering Mechanics 17.2 (2002): 167-176.

35. Hu, Z., and Du, X., 2012, "Reliability Analysis for Hydrokinetic Turbine Blades," Renewable Energy, 48, pp. 251-262 31005-1, 2011. 